stimulating factor (GM-CSF) significantly enhanced IFN- $\lambda 1 / 3$ production by $2-5$ fold. In pDC-NK cell co-cultures from SLE patients, IFN- $\alpha 2 b$ and GM-CSF increased the proportion of RNA-IC responding IFN- $\lambda 1 / 3$ producing individuals from $9 \%$ to $36 \%$. Hydroxychloroquine as well as an interleukin receptor 1 associated kinase 4 inhibitor (IRAK4i) significantly inhibited the RNA-IC-triggered IFN- $\lambda 1 / 3$ production by $\mathrm{pDCs}$ and pDC-NK cell co-cultures by $>90 \%$.

Conclusions Type III IFN production in a small subset of pDCs can be triggered by RNA containing IC, enhanced by NK cells and several pro-inflammatory cytokines, and inhibited by blocking the TLR-MyD88 pathway, resembling the regulation of type I IFN. Thus, our results support a contributing role for both type I and type III IFN in SLE, which needs to be considered when targeting the IFN system in this disease.

\section{P97 DEFICIENCY OF MARGINAL-ZONE B CELLS IN PERIPHERAL BLOOD OF SLE PATIENTS IN CLINICAL REMISSION OR LOW DISEASE ACTIVITY STATE IN A LONG-TERM STUDY}

'Zbynek Hrncir, ${ }^{2}$ Doris Vokurkova, ${ }^{2}$ Marcela Drahosova, ${ }^{1}$ Tomas Soukup. ${ }^{1} 2 n d$ Dept. of Internal Medicine, University Hospital, Hradec Králové; ' 2 Dept. of Immunology and Allergy, University Hospital, Hradec Králové, Czech Republic

10.1136/lupus-2020-eurolupus. 141

Purpose Deficiency of marginal-zone B cells was observed in peripheral blood $(\mathrm{PB})$ of SLE pts in clinical remission or low disease activity $(\mathrm{LDA})^{1}$. Goal of the prospective, comparative, long-term study is follow-up of this phenomenon.

Methods Forty five adult SLE (ACR/1982, updated 1997) pts in complete remission or $\mathrm{LDA}^{2}$ and 10 age- and sex-matched healthy controls (HC) were enrolled in ,month 0', and SLE also after twelve-months (,month 12') and 36-months (, month 36') period; overlap syndromes, infection, renal failure and monoclonal gammopathy in SLE were excluded. The DuraClone IM panel (Beckman Coulter) was used to identify $\mathrm{CD}_{19}{ }^{+} \mathrm{CD}_{27}{ }^{+} \operatorname{IgM}^{+}$B cell subpopulation in $\mathrm{PB}$ samples by flow cytometry navios (Beckman Coulter) with software analysis using Kaluza version 1.2.; data obtained were expressed in relative $\%$ of $\mathrm{PB}$ lymphocytes and absolute values $\times 10^{6} / \mathrm{L}$, and processed using Medcalc-Statistical Software programme.

Results Significant differences ( $p=0.002-<0.001)$ were obtained between absolute values of $\mathrm{CD} 19^{+} \mathrm{CD} 27^{+} \mathrm{IgM}^{+} \mathrm{B}$ cells in HC (median 31.36, 95\% CI 21.49 - 63.35) and SLE „month 0' (median 13.17, 95\% CI 7.87 - 17.09), SLE , month 12' (median 10.56, 95\% CI 7.24 - 16.04), and SLE ,, month 36' (median 9.66, 95\% CI 7.22 - 13.21), but not between values obtained in SLE ,,month 0', ,month 12' and „month 36' ( $p>0.05)$; not significant differences were found using analysis according to relative $\%$ of $\mathrm{B}$ cells under study $(\mathrm{p}>0.05)$.

Conclusion Data obtained demonstrated a long-term deficiency of marginal-zone B cells in PB of SLE pts in complete remission or LDA; susceptibility to infection should be supposed, but further studies are necessary.

Acknowledgement Research project PROGRES Q40-15, Charles Univ. Faculty of Medicine, Hradec Králové, Czech Republic.

\section{REFERENCES}

1. Hrncir Z, et al. Clin Exper Rheumatol 2016:34(S99);S-63.

2. Fanouriakis A, et al. Ann Rheum Dis 2019;78;736-745.

\section{P98 NEUTROPHILS IN LUPUS: A NEW PHENOTYPE}

${ }^{1}$ Sandrine Huot, ${ }^{1}$ Cynthia Laflamme, ${ }^{1}$ Martin Pelletier, ${ }^{1}$ Philippe Tessier, ${ }^{1}$ Eric Boilard, ${ }^{2}$ Paul R Fortin, 'Marc Pouliot. 'Dept. of Microbiology and Immunology, Université Laval, Québec; ${ }^{2}$ Dept. of Medicine, Université Laval, Québec, Canada

\subsection{6/lupus-2020-eurolupus. 142}

Background Polymorphonuclear neutrophils (PMNs) with irregular properties have been reported in patients with lupus and compelling evidence implicates them as a source of self-antigens. PMNs are the most abundant circulating leukocytes in human blood; defining their implication either as inherently defective players or as cells affected by external factors (e.g. autoantibodies, immune complexes and type I interferon) is of interest. We propose that elements present in the blood of patients with lupus affect neutrophil function/viability, in a NETosis-independent fashion.

Methods PMNs were isolated from venous blood of healthy volunteers and incubated with serum from normal subjects or from patients with lupus. Apoptosis and necrosis were assessed in real-time by cell surface exposure of phosphatidylserine (PS) and loss of plasma membrane integrity, respectively. Serums' analytes measurements were made by Luminex and ELISA.

Results Serums from patients with lupus caused a transient increase in PS exposure on the outer leaflet of PMN plasma membrane, in a significantly more intense fashion than serums from normal individuals. This peculiar phenomenon, which does not have characteristics of classic apoptosis, correlated with the Systemic Lupus Erythematosus Disease Activity Index (SLEDAI), in contrast to any of the following serum analytes: cytokines, chemokines, circulating growth factors, HMGB1, S100A8 and A9 proteins, complement components C3 and C4, immunoglobulins (IgA, IgG, IgM) and leukocyte counts. However, the transient PS increase was abolished by the decomplementation of the serums or by blocking the $\mathrm{Fc}$ receptors on PMNs' surface.

Conclusions Healthy PMNs are affected by the serum of patients with lupus. The transient PS exposure on the outer leaflet of the cellular membrane constitutes a new phenotype directly linked with factors that are at play in the blood of patients. Ongoing studies are looking at the nature of this new phenotype and how it links with PMNs being a potential source of self-antigens and/or as players unduly activated in lupus.

\section{P99 EFFECTOR DN2 B CELLS ARE EXPANDED IN A MIXED ANCESTRY COLOMBIAN SLE PATIENT POPULATION}

${ }^{1}$ Carolina Hurtado, ${ }^{2}$ Diego Rojas-Gualdron, ${ }^{3}$ Ricardo Pineda, ${ }^{4}$ Scott Jenks, ${ }^{5}$ Gloria Vasquez, ${ }^{4}$ Iñaki Sanz. ${ }^{1}$ School of Graduate Studies and School of Medicine, CES University, Medellin, Colombia; ${ }^{2}$ School of Medicine, CES University, Medellin, Colombia; ${ }^{3}$ Group of Clinical Information, Artmedica IPS, Medellin, Colombia; ${ }^{4}$ Dept. of Medicine, Division of Rheumatology, Lowance Center for Human Immunology, Emory University, Atlanta, USA; ${ }^{5}$ Grupo de inmunología celular e inmunogenética, Universidad de Antioquia, Medellin, Colombia

\subsection{6/lupus-2020-eurolupus. 143}

Background Double Negative (DN) B lymphocytes are expanded in Systemic Lupus Erythematosus (SLE). Specifically, DN2 cells are a DN subset recently characterized and are functionally plasmablasts precursors. DN2 frequency is higher in SLE patients of African American ancestry and is associated 